\title{
Ergasilus yumaricus sp. n. (COPEPODA: ERGASILIDAE) DAS BRÂNQUIAS DE Pygocentrus nattereri (KNER, 1860), Serrasalmus rhombeus (LINNAEUS, 1819) E Pristobrycon eigenmanni (NORMAN, 1929) (CHARACIFORMES: SERRASALMIDAE) DA AMAZÔNIA BRASILEIRA.
}

\author{
José Celso O. MALTA ${ }^{1}$, Angela M. VARELLA ${ }^{1}$
}

RESUMO - Ergasilus yumaricus sp. n. (Copepoda, Poecilostomatoida, Ergasilidae) é proposta. Os espécimens foram coletados dos filamentos branquiais de Pygocentrus nattereri, Serrasalmus rhombeus e Pristobrycon eigenmanni do Rio Guaporé próximo à Pimenteiras, rios Guaporé e Mamoré próximo à Surprêsa, rio Jiparaná próximo à Jiparaná e Pacaás Novos próximo à GuajaráMirim, Estado de Rondônia, Brasil. A nova espécie tem uma seta forte, pectinada e falciforme no primeiro exopodito, indicando uma relação com outras seis espécies amazônicas. Esta espécie difere das outras na forma do corpo, ornamentações das pernas, antenas e urossomitos.

Palavras chaves: Copepoda, Ergasilus yumarikus sp. n., Amazônia, Serrasalmidae, Rondônia. Ergasilus yumaricus sp. n. (Copepoda: Ergasilidae) from the Gills of Pygocentrus nattereri (Kner, 1860), Serrasalmus rhombeus (Linnaeus, 1819) and Pristobrycon eigenmanni (Norman, 1929) (Characiformes: Serrasalmidae) from Brazilian Amazon.

ABSTRACT-Ergasilus_yumarikus sp. n. (Copepoda, Poecilostomatoida, Ergasilidae) is proposed. The specimens were collected from the gill filaments of the freshwater fishes Pygocentrus nattereri, Serrasalmus rhombeus and Pristobrycon eigenmanni from Guaporé River near Pimenteiras, Guaporé and Mamoré Rivers, near Surpresa, Jiparaná River near Jiparaná and Pacaás Novos River near Guajará-Mirim, Rondônia state, Brazil. The new species has a falciform pectinate seta on the first exopod that indicates relationship to six previously described Amazonian species. This species differs from others in the shape of body, ornamentation of the legs, antennas and urosomites.

Key-words: Copepoda, Ergasilidae, Ergasilus yumarikus sp. n., Amazon, Serrasalmidae, Rondônia.

\section{INTRODUÇÃO}

Atualmente a fauna de copépodos neotropicais, do gênero Ergasilus Nordmann, 1832, é composta por 26 espécies. Destas, 11 (42\%) são marinhas e 15 (58\%) de água doce. Ocorrem principalmente nas brânquias, 23 espécies (88\%), de teleósteos. Uma única espécie foi coletada sobre as nadadeiras (4\%), E.euripedesi Montú, 1980, parasitava as nadadeiras peitorais, dorsais e caudais de Brevoortia plectinata (Jenyns, 1842); Micropogonias furnieri (Desmarest, 1823); Lycengraulis grossidens (Agassiz,1829) e Gobiesox sp., capturados na lagoa dos Patos, estuário do Estado do Rio Grande do Sul, Brasil (MONTU, 1980).

E. hypophthalmi Boeger, Martins \& Thatcher 1993 é a única espécie do gênero coletada nos rastros branquiais (4\%), parasitava duas espécies de siluriformes planctívoros Hypophthalmus edentatus Spix, 1829 e H. fimbriatus Kner, 1857 capturados na foz do Rio Negro, próximo à Manaus, no Estado do Amazonas, Brasil, (BOEGER et al. 1993).

O único exemplo de copépodo

I Departamento de Biologia Aquática, Instituto Nacional de Pesquisas da Amazônia, Caixa Postal 478, Manaus, Amazonas, Brasil, CEP 69011-970. 
do gênero Ergasilus parasitando elasmobrânquio (Sphyrna zigaena) é $E$. myctarothes (Wilson, 1913) coletado em Montego Bay, Jamaica. Também é o primeiro registro deste gênero ocorrendo nas fossas nasais (4\%) (WILSON, 1913).

Do Brasil são conhecidas 18 espécies $(69 \%), 16(61 \%)$ são endêmicas e 13 (50\%) foram descritas e coletadas na região Amazônica. Este trabalho descreve a $27^{\mathrm{a}}$ espécie do gênero Ergasilus da Região Neotropical, a $23^{\mathrm{a}}$ da América do Sul, a $17^{\mathrm{a}}$ do Brasil e a $14^{\text {a }}$ da região Amazonica.

\section{Material e Métodos}

O material foi coletado no Estado de Rondônia, região noroeste do Brasil, durante o período de 28 de novembro de 1983 a 25 de setembro 1985 . Os peixes foram identificados, pesados e medidos. As brânquias e vísceras foram removidas e fixadas em formol $10 \%$. Os copépodos foram retirados dos filamentos branquiais utilizando finos estiletes, microscópio estereoscópio e transferidos para formol 5\%. Lâminas permanentes, com montagem total dos copépodos, foram preparadas usando o método de Thatcher, denominado "HYP" (publicado em Monoculus n.15 de novembro de 1987). Cada indivíduo foi retirado da soluçäo aquosa (formol $5 \%$ ) e colocado em álcool $70 \%$. A seguir, colocado em solução corante, Eosina e Orange-G. Posteriormente colocado em fenol e em seguida em salicilato de metila. Finalmente, foi montado em bálsamo do Canadá entre lâmina e lamínula e colocado em estufa a $70^{\circ} \mathrm{C}$.

Os desenhos foram feitos com o auxílio de câmara clara. As medidas foram obtidas com uma ocular micrométrica e expressas em micrômetros, são dadas as amplitudes seguidas pela média entre parênteses.

Os peixes foram depositados na Coleção Ictiológica do Instituto Nacional de Pesquisas da Amazônia, em Manaus. Os tipos foram depositados na Coleção de Invertebrados do Instituto Nacional de Pesquisas da Amazônia (INPA-CR), Manaus, Amazonas e Museu de Zoologia da Universidade de São Paulo (MZUSP), São Paulo, São Paulo.

Abreviaturas: P1, P2, P3, P4, P5 = primeiro ao quinto pares de pernas.

\section{Ergasilus yumaricus sp. n.}

\section{(Figs 1-11)}

Holótipo: fêmea (INPA-CR 622), dos filamentos branquiais de Pygocentrus nattereri do rio Guaporé próximo a Pimenteiras ( $13^{\circ} 20^{\prime} \mathrm{S}$ e $61^{\circ} 4^{\prime} \mathrm{W}$ ), Estado de Rondônia, Brasil, 28-XI-1984 em lâmina. Parátipos: 17 fêmeas (INPACR 623 a - r) e 3 fêmeas (MZUSP a - c), 4 da localidade tipo, 28-XI-1984; 3 do rio Guaporé, 21-IX-1985 e 3 do rio Mamoré, 24-IX-1985, próximos à Surpresa $\left(11^{\circ} 52^{\prime} \mathrm{S}\right.$ e $\left.64^{\circ} 56^{\prime} \mathrm{W}\right)$; 1 fêmea do rio Pacaás Novos, próximo à Guajará-Mirim $\left(10^{\circ} 40^{\prime} \mathrm{S}\right.$ e $65^{\circ}$ 14 'W) 28-XI-1983; todas do hospedeiro tipo; 2 fêmeas dos filamentos branquiais de Serrasalmus rhombeus da localidade tipo, 28-XI1984; 7 fêmeas de Serrasalmus eigenmanni da localidade tipo, 28-XI1984; todas em lâminas. 110 fêmeas (INPA-CR 624 a - d) e 5 fêmeas 


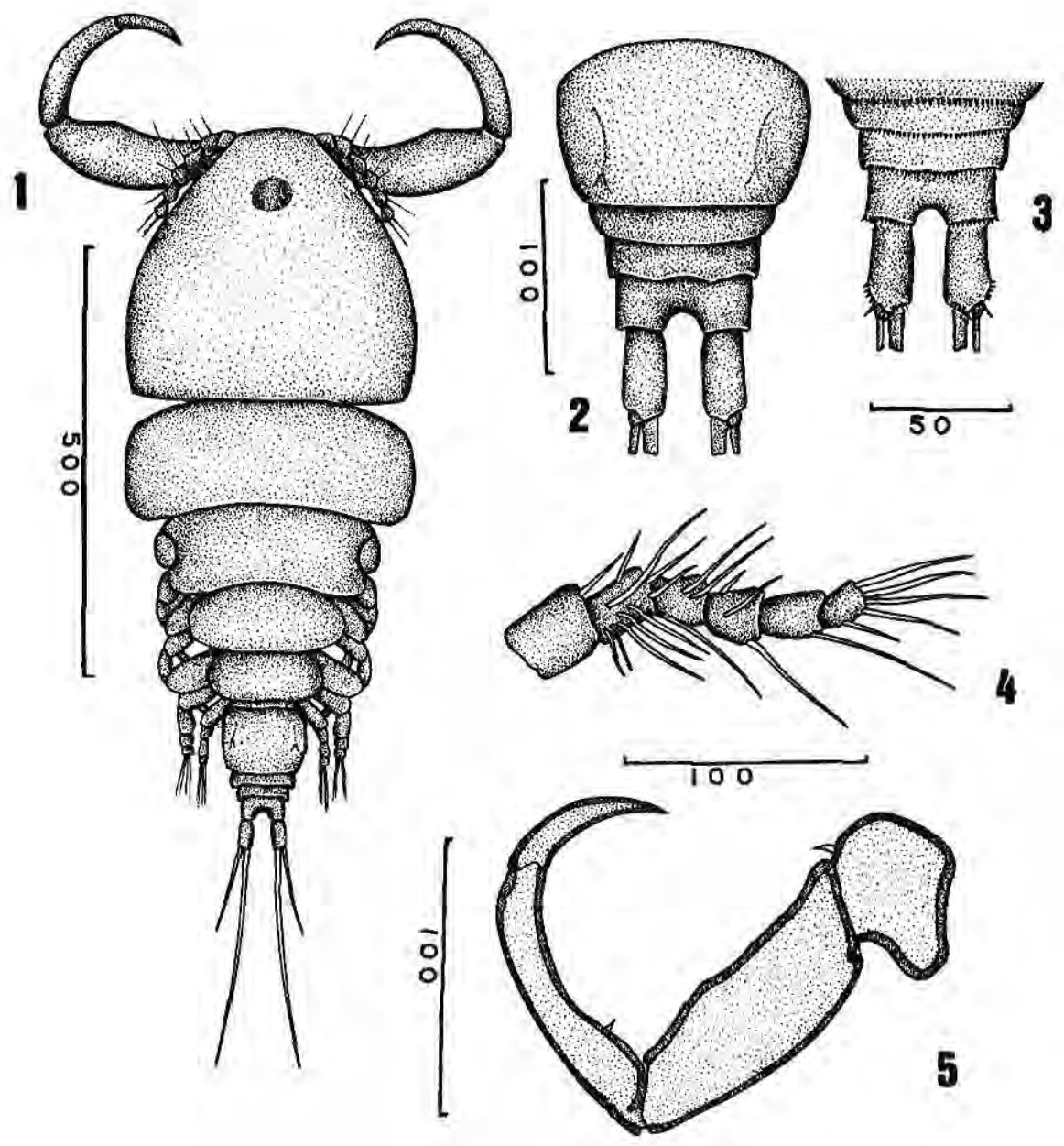

Figuras 1-5. Ergasilus yumarikus (fêmea) sp. n. 1 - corpo inteiro (vista dorsal), 2 - somito genital duplo, urossomitos $3,4,5$ (somito anal) e ramos caudais (vista dorsal). 3 - margem posterior do somito genital duplo, urossomitos $3,4,5$ (somito anal) e ramos caudais (vistas ventral). 4 - antênula. 5 - antena. As medidas das escalas estão em micrômetros. 

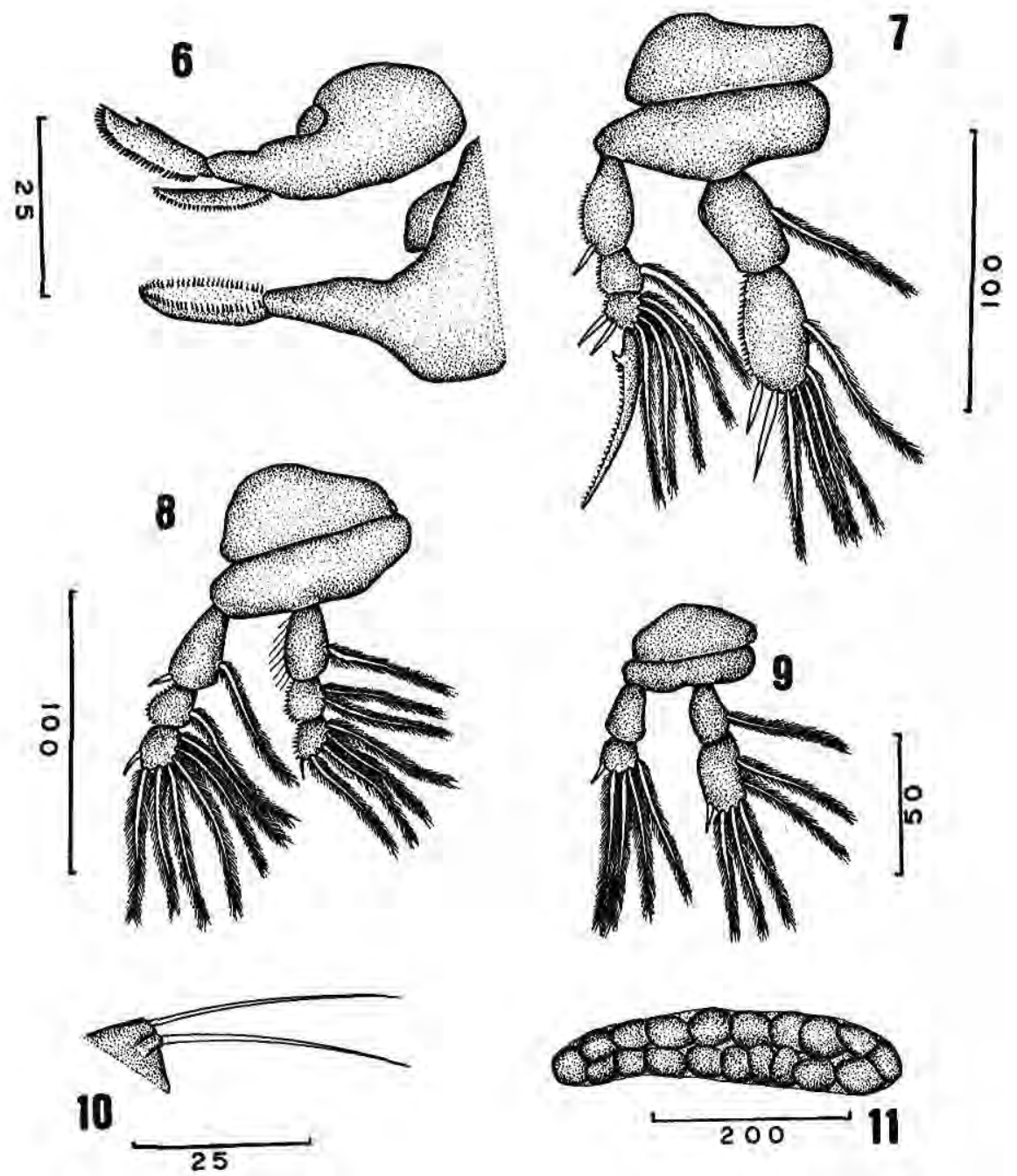

Fugurs 6-11. Ergasilus yumarikus sp. n. (fêmea) 6 - peças bucais. 7 - perna 1 (P1) 8 - perna 2 (P2) $=$ perna 3 (P3). 9 - perna 4 (P4) 10 - perna 5 (P5). 11 - saco de ovos. As medidas das escalas estão em micrômetros. 
(MZUSP - 10447): 39 fêmeas da localidade tipo, 28-XI-1984; 3 fêmeas do rio Guaporé 21-IX-1985 e 4 fêmeas do rio Mamoré 24-XI-1985, próximos à Surpresa; todas do hospedeiro tipo; 69 dos filamentos branquiais de $P$ eigenmanni e da localidade tipo 28-XI-1984, todas em formol $5 \%$. Todo material coletado por J. C. O. Malta.

\section{Fêmea:}

Corpo (Fig. 1) robusto, alguns exemplares com pigmentação "spectrum violet" (cor 72 (SMITHE, 1975)) c'stribuida aleatoriamente no cefalossomo e pernas, comprimento total 577-669 (627). Prossomo mais largo que o urossomo, maior largura do corpo 235306(278).

Cefalossomo (Fig.1) subtriangular, margem anterior reta, alarga-se até o terço final do onde tem a maior largura, afinase ligeiramente formando a segmentaçäo com o primeiro somito pedígero, margens laterais arredondadas. Olho pigmentado na maioria dos exemplares, "smalt blue" (cor 70 (SMITHE, 1975). Metassomo (Fig. 1) com cinco somitos pedígeros (somitos do corpo 2, 3, 4, 5 e 6), todos mais largos que compridos, sub- retangulares, claramente separados e decrescendo de largura gradualmente em direção posterior.

Urossomo (Figs. 1, 2 e 3) cerca de um terço do comprimento do prossomo. Somito $6 \mathrm{com}$ as pernas natatórias rudimentares. Demais urossomitos com uma fileira de pequenos espinhos na margem posterior ventral. Somito genital duplo (Figs. 1, 2 e 3) mais largo que comprido, comprimento 45-60(51), largura 77-87(81), margens laterais arredondadas. Urossomito 3 comprimento 10-17(13), largura 50-65(55); urossomito 4 comprimento 12-15(12), largura 42-52(47); urossomito 5 (somito anal) comprimento 15-25(19), largura 37-47(42). Ramos caudais (Figs. 1, 2 e 3 ) sub-retangulares, comprimento 22-37(32), largura 3242(34), equipados com uma seta longa (comprimento 174-274(230)) uma curta, duas setas reduzidas e com as margens posteriores ventrais com uma fileira de pequenos espinhos.

Antênula (Fig. 4) comprimento 125 - 147(134) e maior largura 22-30(26), cilíndrica, com seis segmentos, carregando 22 setas simples e com a fórmula setal: 1 - 9-3 - 3 - 1 - 5. Antena (Fig. 5) com três segmentos. Segmento 1 o menor, comprimento 55-85(66), largura 47-75(58), com um espinho na região terminal; segmento 2 o maior e o mais robusto, comprimento 110-150(133), largura 4255(49), com uma suave elevação na região mediana da margem interna; segmento 3 o mais fino, comprimento $120-140$ (127) e largura 27-37(33), com um pequeno espinho na margem proximal interna porção distal curva; processo distal é uma forte garra curva, comprimento 82-100(88) e largura 15-20(18). A relação entre os segmentos incluindo a garra é $1,0: 2,0$ : $1,8: 1,2$.

Peças bucais (Fig. 6): mandíbula longa, falciforme, com uma base robusta, larga na região proximal, na região mediana sua largura reduz à metade; na margem posterior, origina-se o palpo mandibular, falciforme e com uma série de minúsculos dentes na margem posterior, o processo basal continua, afila-se e distalmente forma uma lâmina falciforme, com uma pequena reentrância e um dentículo na margem anterior e com 
minúsculos dentes na margem posterior. Maxílula pequena, em forma de placa e sem ornamentaçöes. Maxila bi-segmentada, processo proximal robusto, largo, sem ornamentaçöes e fortemente preso ao cefalossomo, processo distal alongado, base mais larga, afila-se suavemente, terminaImente com pequenas setas espiniformes circundando as margens anteriores. Maxilípedes ausentes.

Pernas (Figs. 7 - 10) providas de setas plumosas. P1 (Fig. 7), todos os segmentos com uma fileira de pequenos espinhos na margem externa, exceto o primeiro segmento do endopodito. Endopodito com dois segmentos e exopodito com três. Segmento 1 do endopodito robusto, sub-retangular, com uma seta e uma pequena regiäo côncava; segmento 2 sub-retangular, robusto com cinco setas e dois fortes espinhos. Segmento 1 do exopodito robusto, o maior, com mais do dobro do tamanho dos demais e com um espinho; segmento 2 com uma seta; segmento 3 com quatro setas, uma seta forte, pectinada, falciforme e dois espinhos.

P2 (Fig. 8) semelhante à P3 com ambos os ramos de três segmentos. Presença de uma pequena depressão circular, na margem interna do coxopodito, de P2 e P3. Segmentos 2 e 3 do endopodito com uma fileira de espinhos. Segmento 1 o maior, cerca de duas vezes maior que os demais, com uma seta e uma fileira de cerdas; segmento 2 com duas setas; segmento 3 com quatro setas e um espinho. Segmento 1 do exopodito o maior, cerca de duas vezes maior que os demais, com uma seta e espinho; segmento 2 com uma seta e uma fileira de pequenos espinhos; segmento $3 \mathrm{com}$ seis setas e um espinho.

P4 (Fig. 9) com ambos os ramos de dois segmentos. Segmento 1 do endopodito sub-retangular e com uma seta; segmento 2 sub-retangular, maior e mais robusto e com cinco setas e um espinho. Segmento 1 do exopodito sem ornamentação; segmento 2 com quatro setas e um espinho.

P5 (Fig. 10) vestigial, consistindo de duas setas simples, do mesmo tamanho, que se originam em uma papila.

Saco ovígero (Fig. 11) com duas séries de ovos que variam, numericamente, de 18 a 26.

\section{Macho: Desconhecido.}

Etimologia: O nome específico deriva do Tupi-Guarani, "yu" espinho; "marika" barriga (abdômen), em alusäo a presença de espinhos nas margens posteriores dos urossomitos.

\section{DISCUSSÃO}

Ergasilus yumarikus sp. n, tem uma seta pectinada, em forma de foice, no segmento terminal do exopodito de $\mathrm{P} 1$, sugerindo afinidades com $E$. bryconis Thatcher, 1981; E. jaraquensis Thatcher \& Robertson, 1981; E. hydrolycus Thatcher, Boeger \& Robertson, 1984; E. callophysus Thatcher \& Boeger, 1984; E. holobryconis Malta \& Varella, 1986; Eurupaensis Malta, 1993 (THATCHER, 1981; THATCHER \& ROBERTSON, 1981; THATCHER \& BOEGER, 1984; THATCHER et al., 1984; MALTA \& VARELLA, 1986; MALTA, 1993.

Ergasilus yumarikus está 
incluído entre as espécies de menor tamanho, seu comprimento total varia de 577 a 669 , situando-se numa faixa muito semelhante à de $E$. leporinidis Thatcher, 1981 (530 a 710), E. colomesus Thatcher \& Boeger, 1981 (540 a 700), E. triangularis Malta, 1993 (524 a 699) e E. holobryconis (525 a 625) (MALTA, 1993; THATCHER, 1981; THATCHER \& BOEGER, 1983).

Comparando-se o comprimento do cefalotórax de $E$. yumarikus com o de outros ergasilídeos, verifica-se que, nesta espécie, corresponde a $50,0 \%$ do comprimento total, proporçäo próxima à encontrada por THATCHER (1984) para $E$. jaraquensis $(51,0 \%)$.

Algumas espécies neotropicais apresentam a extremidade distal, ventral dos urossomitos com uma fileira de pequenos espinhos: E. hydrolycus apresenta no urossomito 5 e nos urópodos; E. pitalicus Thatcher 1984; E.yumarikus e E.hypophthalmi Boeger, Martins \& Thatcher, 1993 apresentam nos urossomitos 2, 3, 4, 5 e urópodos (BOEGER et al, 1993; THATCHER, 1984; THATCHER et al., 1984.).

Somente E. yumarikus e $E$. iheringi Tidd, 1942, entre as espécies Neotropicais, não possuem um espinho no primeiro segmento do exopodito de $\mathrm{P} 4$. Mas apesar disto, $E$. iheringi é a única espécie neotropical que só tem um segmento no exopodito de P4 (TIDD, 1942), todas as demais têm dois.

A combinaçäo destes caracteres aliados à: ornamentação da antena; presença de depressões circulares, uma na margem externa do endopodito de P1 a outra na margem interna do coxopodito de P2 e P3; inserção do primeiro com o segundo somito; formato do corpo; formato das peças bucais; fórmula setal; variação do número de ovos e proporções dos segmentos da antena, são características exclusivas de $E$. yumarikus.

Os índices que estimam o tamanho da população de $E$. yumarikus são: prevalência $30,0 \%$; intensidade entre 1-92 copépodos por peixe: intensidade média $15,0 \mathrm{e}$ abundância 5,0.

\section{Bibliografia citada}

BOEGER, W. A; MARTINS, M.; THATCHER, V, E. 1993. Ergasilus hypophthalmi sp. n. (COPEPODA, POECILOS TOM ATOIDA, ERGASILIDAE) from the gill rakers of the amzonian catfishes, Hypophthalmus edentatus Spix and $H$. fimbriatus Kner. Rev. Brasil. Biol., 53(3):421-424.

MALTA, J. C. O. 1993. Ergasilus urupaensis sp. n. (COPEPODA: ERGASILIDAE) das brânquias de Prochilodus nigricans Agassiz, 1829 (CHARACIFORMES: PROCHILODONTIDAE) da Amazônia Brasileira. Acta Amazônica, 23(4):449-456

MALTA, J. C. O.; VARELLA, A. 1986. Ergasilus holobryconis sp. n. parasita de Holobrycon pesu (Muller \& TroschelL), um peixe da Amazônia Brasileira (COPEPODA: POECILOSTOMATOIDA: ERGASILIDAE). Amazoniana, 10(1):41-48.

MONTU, M. 1980. Parasite copepods of Southern Brazilian fishes I- Ergasilus euripedesi n. sp. (Copepoda: Cyclopidea). Iheringia, 56: 53-62.

SMITHE, F. B. 1975. Naturalist' Collor Guide and Suplement. I. New York. Colors 1$86+229 p p$.

THATCHER, V. E. 1981. Os crustáceos parasitos 
de peixes da Amazônia Brasileira, 2. Ergasilus leporinidis sp. n. (Copepoda: Cyclopoidea) das brânquias de Leporinus fasciatus (Bloch). Acta Amazonica, 11(4): 723-727.

1984. Ergasilus pitalicus, new species (Copepoda: Poecilostomatoida: Ergasilidae) a gill parasite of a cichlid fish from the $\mathrm{Pa}$ cific coast of Colombia. J. Crust. Biol., 4(3):495-501.

THATCHER, V. E.; BOEGER, W. A. 1983. The parasitic crustaceans of fishes from the Brazilian Amazon. 4. Ergasilus colomesus sp. n. (Copepoda: Cyclopoidea) from an omamental fish, Colomesus asellus (Tetraodontidae) and aspects of its patogenicity. Trans. Am. Microsc. Soc., 102(4): 371-379.

1984. The parasitic crustaceans of fishes from the Brazilian Amazon. 9. Ergasilus callophysus sp. n. (Copepoda: Cyclopoidea) from Callophysus macropterus (Lichtenstein). Proc. Helm. Soc. Was., 51(2): 326-330.
THATCHER, V. E.; BOEGER, W. A.; ROBERTSON, B. A. 1984. The parasitic crustaceans of fishes from the Brazilian Amazon. 12. Ergasilus hydrolycus sp. n. (Copepoda: Poecilostomatoida) from Hydrolycus scomberoides (Cuvier). Amazoniana, 8(3): 321-326.

THATCHER, V. E.; ROBERTSON, B. A. 1981. The parasitic crustaceans of fishes from the Brazilian Amazon. 3. Ergasilus jaraquensis $\mathrm{sp}$. $\mathrm{n}$. (Copepoda: Cyclopoidea) from the gills of Semaprochilodus insignis (Schomburgk). Rev. Bras. Biol, 42(3): 515-519.

TIDD, W. M. 1942. A new species of Ergasilus (Parasitic, Copepoda) from a characinid fish of Brazil. Tr. Am. Micr. Soc., 62(1); 62 -65.

WILSON, C. B. 1913. Crustaceans parasites of West Indian fishes and land crabs, with descriptions of new genera and species. Proc. U. S. Nat. Mus., 44:189-277, (34 plates). 